\title{
Primary Intimal Pulmonary Vein Sarcoma with Expansion to the Left Atrium
}

\author{
Aleksandar Nikolić \\ ${ }^{1}$ Cardiac surgeon, Chief of Department of Cardiac Surgery, Clinical Centar of Montenegro, Europe \\ ${ }^{2}$ Resident of cardiac surgery, Department of Cardiac Surgery, Clinical Centar of Montenegro, Europe \\ ${ }^{3}$ Cardiac surgeon, Department of Cardiac Surgery, Clinical Centar of Montenegro, Europe \\ ${ }^{4}$ Internal medicine specialist, Department of Cardiac Surgery, Clinical Centar of Montenegro, Europe \\ ${ }^{5}$ Cardiology specialist, Department of Cardiac Surgery, Clinical Centar of Montenegro, Europe
}

Received: July 11, 2017; Published: July 18, 2017

*Corresponding author: Aleksandar Nikolić, cardiac surgeon, Chief of Department of Cardiac Surgery, Clinical Centar of Montenegro, Montenegro, Europe, Email: draleksandarnikolic@gmail.com

\begin{abstract}
We have recently treated a patient with pulmonary vein sarcoma. It is a 65 year old women who was admitted to the hospital because of the chest pain with propagations down her right arm, followed by suffocation and coughing out content with traces of blood and febrility $>38^{\circ} \mathrm{C}$. The primarily suspected pulmonary embolism was ruled out after the diagnostic heart CT scan and transthoracal and transesophageal echocardiogram which verified the existing of a tumor mass in the left atrium. The patient underwent an urgent surgery and the tumor mass was removed surgically from the upper right pulmonary vein. The path histological diagnoses revealed pulmonary vein intimal sarcoma. Pulmonary vein intimal sarcoma is one of the rarest sarcoma subtypes. To our knowledge, there is only one published case of such pathology in the literature.
\end{abstract}

\section{Introduction}

A 65 year old female patient was admitted in the Emergency Room of Montenegrin Clinical Hospital because of the chest pain with propagations down her right arm, followed by suffocation and coughing out content with traces of blood and febrility $>38^{\circ} \mathrm{C}$. She mentioned the poor physical exercise tolerance with occasional swelling of lower extremities. She was experiencing the discomforts for the previous 10 days [1-3]. The patient had been a smoker for a long time. She was aware of cardiac arrhythmia which is why she had been prescribed an oral anticoagulant therapy. She had been aware of her condition of thrombocytopenia too. Twenty years ago she underwent hysterectomy with bilateral adnexectomy.

During the physical examination the patient was eupnotic while resting, acyanotic, anicteric and afebrile. Her vital signs were: cardiac frequency (FR) 85/min, blood pressure (TA) 100/60 mmHg, respiratory frequency (FR) around $16-18 / \mathrm{min}$, saturation (SO2) around 96\%. Pulmonary auscultation revealed a weakened basal respiratory wheeze with sporadic late inspiratory bilateral basal crackles [4-7]. The auscultation also showed a rhythmical cardiac activity with clear tones and without wheezes. The extremities were without edemas, varicosity or deformities. EKG showed the sinus rhythm of around 85/min, normogram, without signs of ischemia.

\section{Laboratory Test Results}

Se 30...72, CRP 10...160, D-dimer 0,73, PV 14,2, INR 1,2, fibrinogen 81, Le 5,74, Er 3,96, Hgb 122, HCT 0,35, Tr 162. Pulmonary X- ray showed left basal pleural pericardial effusion. Chest CT scan describes a $35 \mathrm{~mm}$ thrombus in the main part of Art. Pulmonalis which extends to the branch for the upper lobe with consequential parenchyma consolidation ventrally bigger than 50 $\mathrm{mm}$, pericardially along with the pericardium almost completely occupying the front FC sinus and dorsally with the lower branch of interlobar incisura up to $30 \mathrm{~mm}$ and medially almost at the very top of the lungs with the size of up to $20 \mathrm{~mm}$ with the complete hypo perfusion of the upper lobe with evidently positive air bronchogram.

Heart CT scan describes in the left atrium a big tumor-like change with the dimensions of $32 \times 24 \mathrm{~mm}, 32 \mathrm{~mm}$ long along which thrombotic masses are evident. The tumor covers both lower left 
pulmonary veins which are dilated and filled with thrombotic masses. Mediastinum contains numerous vein blood vessels which can correspond to obstructing changes at the level of azygos system. Right ventricle is of borderline size, the right ventricular outflow tract is preserved. The signs of pulmonary artery dilatation are evident without any presence of thrombosis. Under the left ventricle an isolated pericardial effusion can be seen in the pericardium. In pleura's posterobasal and anterobasal segments the evidence of atelectasis are evident till the lower lingual. Both apical and lingular segments are peripherally sub-atelectatic. Left basal pleural effusion is also evident.

\section{Conclusion}

Tumor-like change of left atrium with thrombosis of lower left pulmonary veins, peripheral vein plethora and of lingular left lung segment. Pleural and pericardial effusion. TTE shows in the left atrium which is of normal dimension, or to be more precise near the top of left pulmonary veins confluence a fixed non-floating hyperechogenic formation with the dimensions $22 \times 26 \mathrm{~mm}$. The diagnosis is confirmed by TEE which showed a tumor mass in LA with the size of $20 \times 40 \mathrm{~cm}$ and non-homogenous structure which is attached to the top of LP. Within the pre-operating preparation, hemodynamic process was conducted which showed no hemo dynamically significant stenosis on coronary arteries.

General endo tracheal anesthesia was induced and after the establishing of extracorporeal blood flow, with the incision through right atrium and inter atrial septum, tumor jelly-like mass was identified with the diameter of around $30 \mathrm{~mm}$ on the top of left atrium which was obstructing the confluence of lower pulmonary veins, with a long petiole the base of which was attached to the wall of left upper pulmonary vein. The tumor was not in contact with the mitral apparatus structures and there was no need for interventions on the valvular apparatus.

\section{Patho Histological Results}

Macroscopic: Several tissue fragments with the size up to $40 \mathrm{~mm}$ and with softer to medium hard consistency, partly covered in slime.

Microscopic: Tumor tissue made of groups and solid clusters of atypical spindle and oval cells. The nuclei are slightly to moderately polymorphic, oval and hyper chromatic.

A. A moderate number of mitoses - focally between 5 and 10 mitoses on 10 fields of big enlargements.

B. The tumor contains bleeding zones and small necrosis focuses.

C. Hypo cellular slimy degenerated areas were present.

\section{Immuno histo chemical}

Tumor cells are Vimentin and Actin positive; CK, EMA, Desmin, S100, CD 34 and HMB 45 negative. The path histologically analyzed mediastinal lymph node is of preserved structure, without tumor tissue. Path histological diagnosis intimal sarcoma (gradus 2).

\section{Discussion}

Primary neoplasm of big blood vessels (aorta, pulmonary artery and pulmonary veins, v. cava sup and inf.) are extremely rare. The pathogenesis of those tumors is still vague and signs and symptoms are unspecific which makes differential diagnosis difficult and postpones a final diagnosis. Around 400 cases of primary tumors have been mentioned in reference books so far, 30 of them being cases of primary pulmonary vein tumors. The majority of those tumors are leiomyosarcomas and there is one case of myxosarcoma and one case of myxoidfibrosarcoma. These pulmonary vein sarcomas are often located in the upper right and lower left pulmonary veins. To our knowledge this is the second published case of pulmonary vein non-myxioid intimal sarcoma (fibro sarcoma). The biggest group of patients with pulmonary vein primary sarcoma includes 17 cases of leiomyosarcoma. Blood vessel tumors appear on average when people are in their 40 s and they are equally found in both sexes (leiomyosarcomas mildly predominate with females). The most common symptoms like cough, hemoptysis, dyspnea, chest pain and pleural effusions are unspecific. Pulmonary vein sarcomas, especially intimal sarcomas can be misdiagnosed as pulmonary thromboembolism, which was initially the case with our patient, because of the fact that it is rare and because of unclear clinical progress. The consequences can be inadequate treatments like a prolonged anticoagulant therapy or thrombolysis. In the biggest series of pulmonary vein leiomyosarcomas, around $50 \%$ of the cases were diagnosed with the help of CT and/or MRI angiography. It is important here to be mentioned that in majority of such cases the tumor masses are thought to be originating from left atrium. Bronchoscopy and transesophageal echocardiography together with heart CT are important for proper diagnosing and urgent management of those cases.

The difficulties in diagnosing are only one of the aspects related to bad prognosis of primary pulmonary vein tumors, regardless of the subtype. Only two cases were recorded when patients lived three or more years after the treatment of leiomyosarcoma and only one patient lived more than nine months after the treatment of myxosarcoma. Both patients with leiomyosarcoma had a complete surgical resection with clean remaining edges. After the resections there was lots of case of relapsing which makes us conclude there is a need for adjuvant or non-adjuvant therapy. Surgery with adjuvant chemotherapy and radiotherapy can improve short term survival. However the prognosis is not good with the rate of five year survival between 0 and 6 percent.

The ideal management of pulmonary vein sarcomas is still being discussed. Extensive surgical resection is for now considered to be the most optimal solution. For patients with incomplete resection or local relapsing heart transplanting is taken into consideration as a valid solution. Although some authors do not think that chemotherapy and radiotherapy are efficient, some other authors hope that systemic chemo and radio therapy could increase the survival rate and achieving therapy and palliative benefits could avoid potential surgical complications. 


\section{References}

1. Constantine D Mavroudis, Alfred Casillan, Mark Rabbat, Aileen Go, Edward Melian, et al. (2012)"Primary Intimal Sarcoma of the Pulmonary Vein with Estension into the Left Atrium: Case Report. Open Journal of Thoracic Surgery 2(2): 25-28.

2. Zhang Wei-min, Xu Yong, Jiang Zhi-nong, Su Xue-feng, He Chao (2012)" Pulmonary vein sarcoma: a case report". Chin Med J 125(5): 954-956.

3. Na Rae Kirn, Jhingook Kim, Seung Yeon Ha, Joungho Han (2011) " Pulmonary Vascular Sarcomas: Clinicopathologic Analysis of 14 Cases". The Korean Journal of Pathology 45(2): 132-138.

4. Burke AP, Virmani R (1996) Primary cardiac sarcomas in tumors of the heart and great vessels. In: Burke AP, Virmani R, eds. Atlas of tumor pathology ( $3^{\text {rd }}$ ed) Washington. DC: Armed Forces Institute of Pathology 127-169.

5. Burke AP, Virmani R (1993) Sarcomas of the great vessels. A clinicopathologic study. Cancer 71(5): 1761-1773.

6. Gürbüz A, Yetkin U, Yilik L, Ozdemir T, Türk F (2003) A case of leiomyosarcom originating from pulmonary vein, occluding mitral inflow. Heart Lung 32(3): 210-214.

7. Oliai BR, Tazelaar HD, Lloyd RV, Doria MI, Trastek VF (1999) Leiomyosarcoma of the pulmonary veins. Am J Surg Pathol 23(9): 10821088.

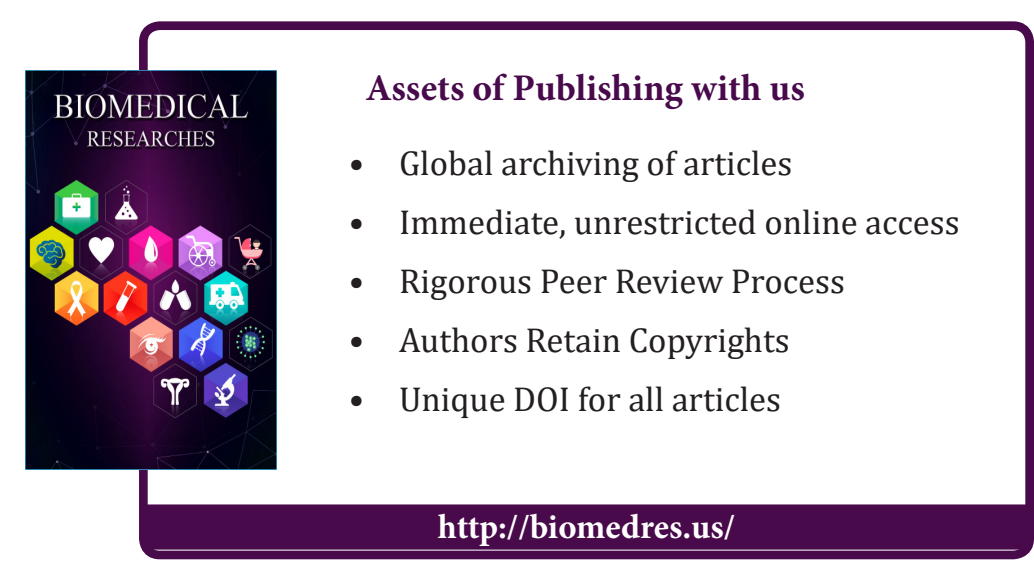

